\title{
The estimated incidence of lactational breast abscess and description of its management by percutaneous aspiration at the Douala General Hospital, Cameroon
}

\author{
Thomas Obinchemti Egbe ${ }^{1,2^{*}}$, Theophile Nana Njamen ${ }^{1,2}$, Henri Essome $e^{3,4}$ and Nicholas Tendongfor ${ }^{1}$
}

\begin{abstract}
Background: Lactational breast abscesses are uncommon in the puerperium but when they do develop, delays in specialist referral may occur especially in resource low settings. There is a dearth of studies regarding lactational breast abscesses in Cameroon. We aimed to estimate the incidence of lactational breast abscess and describe its management by percutaneous aspiration at the Douala General Hospital, Cameroon.

Methods: We conducted an observational prospective study of 25 breastfeeding women at the Douala General Hospital from January 1, 2015, to October 31, 2015. Participants were consenting breastfeeding women who completed a baseline questionnaire after diagnosis of lactational breast abscesses and underwent percutaneous needle aspiration under local anaesthesia. Data were analyzed by using descriptive statistics.

Results: The estimated incidence of lactational breast abscesses was $0.74 \%$ (28/3792). The age range of babies at the onset of breast abscess was 4 to 35 weeks; mean $28.3 \pm 10.85$ weeks. Forty-four per cent of participants underwent three lactational abscess aspirations and in 24 to $28 \%$ of them, it took 8 to 9 days for the abscess to resolve. In $72 \%$ of participants, treatment was with needle aspiration plus flucloxacillin. Seventy-six per cent of participants continued breastfeeding after abscess treatment.

Conclusion: The estimated incidence of lactational breast abscess at the Douala General Hospital is $0.74 \%$. Percutaneous needle aspiration under local anaesthesia is an effective treatment for superficial lactational breast abscesses in most cases with or without ultrasound guidance and should be recommended worldwide as first line treatment. Further research is needed to understand the outcome of local infiltration of antibiotics on the abscess cavity.
\end{abstract}

Keywords: Abscess, Breast, Breastfeeding, Drainage, Lactation, And mastitis

\footnotetext{
* Correspondence: toegbe@gmail.com; obinchemti@yahoo.com

${ }^{1}$ Faculty of Health Sciences, University of Buea, Box 63, Buea, Cameroon

${ }^{2}$ Department of Obstetrics and Gynecology, Douala General Hospital, P.O.

Box 4856, Douala, Cameroon

Full list of author information is available at the end of the article
}

C The Author(s). 2020 Open Access This article is licensed under a Creative Commons Attribution 4.0 International License, which permits use, sharing, adaptation, distribution and reproduction in any medium or format, as long as you give appropriate credit to the original author(s) and the source, provide a link to the Creative Commons licence, and indicate if changes were made. The images or other third party material in this article are included in the article's Creative Commons licence, unless indicated otherwise in a credit line to the material. If material is not included in the article's Creative Commons licence and your intended use is not permitted by statutory regulation or exceeds the permitted use, you will need to obtain permission directly from the copyright holder. To view a copy of this licence, visit http://creativecommons.org/licenses/by/4.0/. The Creative Commons Public Domain Dedication waiver (http://creativecommons.org/publicdomain/zero/1.0/) applies to the data made available in this article, unless otherwise stated in a credit line to the data. 


\section{Background}

Lactational breast abscesses are complications of infectious mastitis and are more frequent among primiparous women. It has been estimated that 0.4 and 3\% of women with mastitis develop a breast abscess [1]. A breast abscess is defined as a localized accumulation of infected fluid in breast tissue [2].

Mastitis usually occurs during the first 6 weeks but can occur at any time during lactation [2, 3]. Besides, the incidence of lactational breast abscesses is reducing over time because of the increasing use of antibiotics and improved breastfeeding practices [3]. The most common causative microorganism is Staphylococcus aureus, and methicillin-resistant Staphylococcus aureus (MRSA) is becoming an increasing problem. Furthermore, other organisms like Escherichia coli and Haemophilus influenza have been identified [2, 4, 5].

Risk factors for a lactational breast abscess include increasing maternal age at delivery, gestational age greater than 41 weeks, mastitis, primiparity, mother employed outside the home and being married, having breastfeeding difficulties in hospital and having cracked nipples [6, 7]. The diagnosis of lactation breast abscess is clinical and confirmed by an ultrasound scan when available $[8,9]$.

Antibiotics and incision and drainage have been viewed as standard therapy in managing lactational breast abscesses. Antibiotics of choice such as flucloxacillin $500 \mathrm{mg}$ four times daily orally, or after antibiotic susceptibility testing may be prescribed especially in case of MRSA. Women who are allergic to penicillin may be prescribed Cephalexin but clindamycin is suggested for cases of severe penicillin hypersensitivity. It is noteworthy that MRSA should be presumed to be resistant to treatment with macrolides and quinolones, regardless of susceptibility testing results $[2,10]$. More recently, however, there has been an emergence of studies favouring treatment of lactational abscesses with ultrasoundguided needle aspiration, which is considered a less invasive technique that causes less scarring, does not affect breastfeeding, does not require general anesthesia or hospitalization and is less expensive than incision and drainage (I \& D). Although I \& D has the advantage of breaking down the loculi, if the procedure is carried out under general anesthesia it will also involve hospitalization and regular dressings. This could cause considerable distress to both mother and baby during what can already be a difficult and busy time. Also, I \& $\mathrm{D}$ is associated with a prolonged healing time, regular dressings, difficulties in breastfeeding, and the possibility of an unsatisfactory cosmetic result and breastfeeding experience $[8,10,11]$. There is a dearth of studies that describe the clinical management of lactational breast abscess in Cameroon.
In Cameroon, the 2011 Demographic Health Survey reported that while $97 \%$ of mothers initiate breastfeeding, only $31 \%$ of mothers practice exclusive breastfeeding by $0-1$ month, $22 \%$ by $2-3$ monthsand $10 \%$ by $4-5$ months. The median duration of breastfeeding is 16.5 months [12]. Other Cameroon studies have reported $17.3 \%$ [13] and 20\% EBF by 6 months and average breastfeeding time of 15.24 months [14].

This study aimed to estimate the incidence of lactational breast abscess and describe its management by percutaneous aspiration at the Douala General Hospital, Cameroon.

\section{Methods}

\section{Participants and setting}

We conducted an observational prospective study from January 1 to October 31, 2015, at the Douala General Hospital (DGH). The DGH is a tertiary care referral institution in Douala with a neonatal intensive care unit and conducts about 1250 births per year. Data was collected from breastfeeding mothers who gave birth at the DGH and returned later because of a breast condition and those referred from other health facilities; Laquintinie Hospital Douala (LHD), a secondary care centre with 2750 births annually and Cite Des Palmier District Hospital Douala (CPHD), a primary care centre with 550 births per year.

\section{Study population and sampling}

We included in the study consenting breastfeeding mothers with normal vaginal delivery of healthy singleton babies at term. The babies had to be normal (normal birth weights and devoid of congenital malformations). Women must have chosen to breastfeed (exclusively or partially). Participants were consecutively enrolled in the study in a non-discriminatory manner as they came to the outpatient department of the Obstetrics and Gynecology Department of the DGH after signing an informed consent form. They were interviewed in English, French or Pidgin English according to the mother's preference.

Excluded from the study were women with other medical conditions, (diabetes, HIV, renal failure and suspected malignancy), patients with imminent necrosis of skin overlying breast abscess, those who had chosen not to breastfeed or those who did not provide consent.

The following variables were recorded on a pretested interviewer-administered questionnaire: maternal age, gravidity or parity, marital status, educational background, and employment status and smoking history were obtained. Data concerning the place of antenatal care, previous births, whether or not there was nipple damage or pain, history of mastitis/breast abscess in multiparous women, breastfeeding experiences and 
counselling from healthcare providers. Lactational breast abscesses were diagnosed clinically by the following signs: breast tenderness/pain, redness of any part of the breast, breast lump, and high temperature. Most participants were first diagnosed with breast abscesses more than 3 weeks after giving birth. Milk culture or ultrasonography was not accessible due to cost. However, the clinical findings were disclosed to the participants and we noted the number of breast aspirations, antimicrobial treatment, duration of follow-up, and duration of breastfeeding.

Written informed consent was obtained from the participants and we complied with the Helsinki declaration throughout the study [15].

\section{Lactational breast abscess aspiration technique}

We performed the procedure on an outpatient basis. A 14-gauge needle mounted on a $20 \mathrm{~mL}$ syringe was used for aspiration without ultrasonographic guidance. Local anesthesia (lidocaine 2\%) was infiltrated at the puncture site using a 29 -gauge needle, then a $21 \mathrm{G}$ and finally the $14 \mathrm{G}$ needle. We selected the entry site after cleansing with chlorhexidine to avoid the area of skin thinning if present, where an abscess may drain spontaneously. We aspirated the abscess thoroughly and irrigated the cavity with saline until the aspirate became clear (Fig. 1). This method enabled loculations to be disrupted. We did not send the aspirate to the laboratory for culture because of elevated cost. In some cases, we injected $1 \mathrm{~g}$ of ceftriaxone directly into the abscess cavity. Oral flucloxacillin, $500 \mathrm{mg}$ four times a day or erythromycin propionate in case of hypersensitivity of penicillin, was also administered for 10-14 days to these patients. Follow-up of patients was by clinical examination and dressing every 2 days for 1 week and every week for two consecutive weeks,

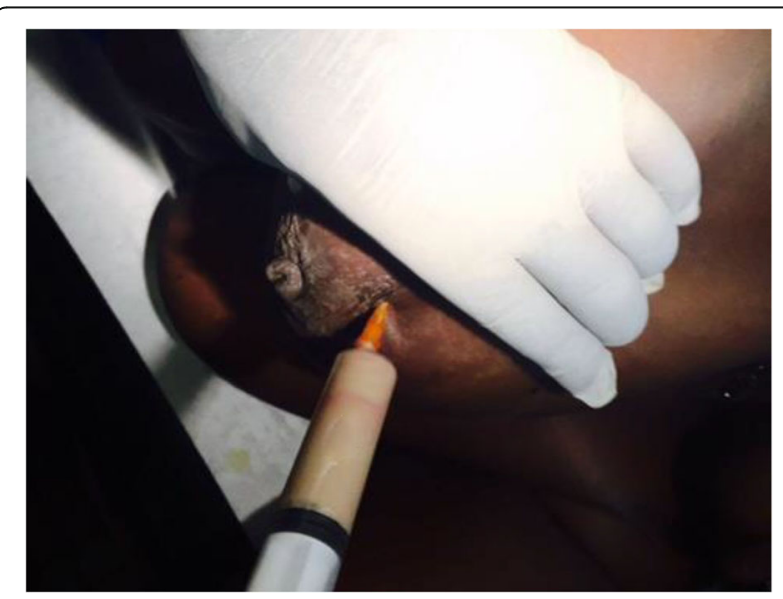

Fig. 1 Lactational breast abscess. Aspiration of pus with a 14-gauge needle mounted on a $20 \mathrm{~mL}$ syringe and further aspirations were performed as deemed necessary. We discontinued follow-up when there was no clinical evidence of inflammation and we encouraged patients to continue breastfeeding.

\section{Data management and statistical analysis}

The filled questionnaires were checked for completeness, coded, entered into a Microsoft Excel spreadsheet and exported to the statistical package for the social sciences (SPSS) version 20 for analysis. Data from questionnaires were double entered and merged to check for data entry errors. Categorical variables were summarized as counts and percentages whilst continuous variables were summarized as means and standard deviations.

\section{Types of intervention}

1. Lactational abscess aspiration with oral flucloxacillin

2. Lactational abscess aspiration with oral erythromycin

3. Lactational abscess aspiration with oral flucloxacillin and instillation of ceftriaxone

\section{Outcome measures}

1. Time to resolution of a breast abscess (no recurrence of abscess or need for further intervention). Time was defined in this study as time of presentation for care.

2. Duration of continuation of breastfeeding

This was defined as the totality of duration of breastfeeding both before and after abscess treatment as reported by the participants.

Data were analyzed by using descriptive statistics.

\section{Results}

During the period of study 4550 live births were recorded in the catchment health facilities (DGH, LHD, $\mathrm{CPDH})$ per year. However, the study period was 10 months giving a total of $(10 / 12$ of $4550=3792)$ live births. Twenty-eight patients were diagnosed with lactational breast abscess during the study period. Therefore, the estimated incidence of lactational breast abscess in this study period was $0.74 \%$ (28/3792). Twenty-five (89.3\%) of the 28 patients diagnosed with a lactational breast abscess consented to involvement in and completed the study (Fig. 2).

Table 1 shows that participant's ages ranged from 17 to 43 years with a mean age of $29.7 \pm 7.1$ years. The duration of breastfeeding ranged from 12 to 104 weeks with a mean of $45.4 \pm 27.8$ weeks. The age of babies at the 


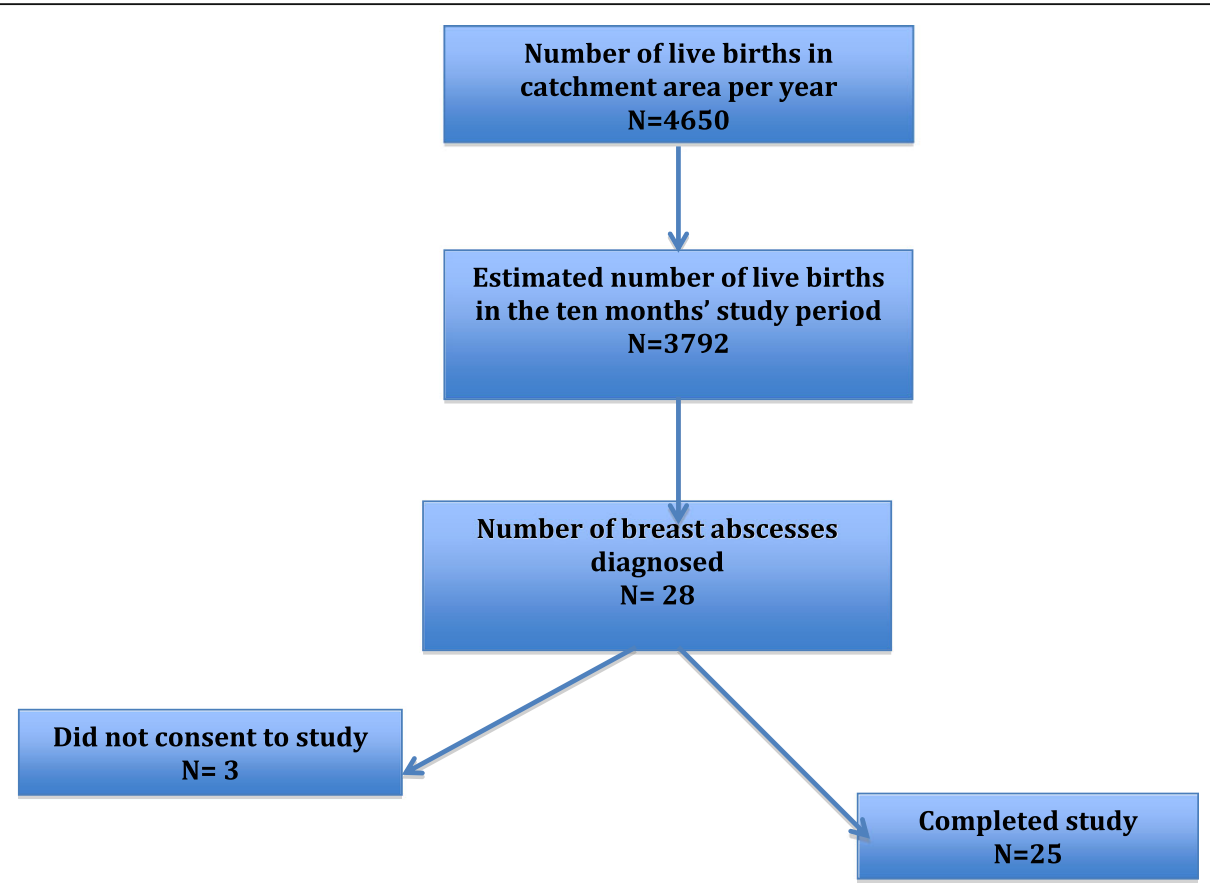

Fig. 2 Flow diagram of lactational breast abscess

onset of the breast abscess ranged between 4 and 35 weeks with a mean age of $28.33 \pm 10.85$ weeks.

As shown in Tables 2, 56\% of participants were less than 30 years old, and most participants were married and multiparous. The highest level of educational attainment for $80 \%$ of the participants was secondary school and $12 \%$ were unemployed.

Table 3 shows that $44 \%$ of participants received three lactational abscess aspirations and it took 8 to 9 days for the abscess to resolve in 24 to $28 \%$ of women respectively. Seventy-six per cent of participants continued breastfeeding after abscess treatment. Furthermore, only $16 \%$ of participants had prior breastfeeding counselling. Moreover, 29\% of multiparous women had a history of mastitis and $72 \%$ of participants were treated with oral flucloxacillin and abscess aspiration.

Table 1 Descriptive statistics $(N=25)$

\begin{tabular}{ll}
\hline Characteristics & Frequency or Value \\
\hline Maternal Age (Years) & 29.72 \\
Mean & 7.07 (17-43) \\
Standard deviation (range) & 28.33 \\
Baby's age at the onset of breast abscess (Weeks) \\
Mean & 10.85 (4-35) \\
Standard deviation (range) & \\
Duration of breastfeeding (Weeks) & 45.36 \\
Mean & $27.8(12-104)$ \\
\hline
\end{tabular}

\section{Discussion}

This study aimed to estimate the incidence of lactational breast abscess and describe its management by percutaneous aspiration at the Douala General Hospital, Cameroon. The estimated incidence of lactational breast abscess is $0.74 \%(28 / 3792)$. This incidence is consistent with the $0.4 \%$ (95\% CI $0.14,0.98)$ reported by an Australian cohort study [1]. Furthermore, literature reports that

Table 2 Demographic characteristics of the participants $(N=25)$

\begin{tabular}{lll}
\hline Characteristics & Frequency & Percentage \\
\hline Age distribution (years) & 14 & 56 \\
$15-30$ & 11 & 44 \\
$>30$ & & \\
Parity & 17 & 68 \\
$\quad$ Multiparity & 8 & 32 \\
Primiparity & & \\
Marital status & 17 & 68 \\
Married & 8 & 32 \\
Single & & \\
Level of education & 20 & 80 \\
Secondary & 3 & 12 \\
Primary & 2 & 8 \\
University & & 88 \\
Employment status & 22 & 12 \\
Yes & 3 & \\
No & & \\
\hline & &
\end{tabular}


Table 3 Clinical characteristics of participants $(N=25)$

\begin{tabular}{|c|c|c|}
\hline Characteristics & Frequency & Percentage \\
\hline \multicolumn{3}{|l|}{ Number of aspirations } \\
\hline 3 & 11 & 44 \\
\hline 2 & 8 & 32 \\
\hline 1 & 6 & 24 \\
\hline \multicolumn{3}{|l|}{ Duration of follow-up (resolution time) } \\
\hline 6 & 4 & 16 \\
\hline 7 & 4 & 16 \\
\hline 8 & 6 & 24 \\
\hline 9 & 7 & 28 \\
\hline 10 & 4 & 16 \\
\hline \multicolumn{3}{|l|}{ Continued breastfeeding } \\
\hline No & 6 & 24 \\
\hline Yes & 19 & 76 \\
\hline \multicolumn{3}{|l|}{ Breastfeeding counseling received } \\
\hline No & 21 & 84 \\
\hline Yes & 4 & 16 \\
\hline \multicolumn{3}{|l|}{ Place of antenatal care } \\
\hline Not in the Douala General Hospital & 16 & 56 \\
\hline Others & 7 & 24 \\
\hline Douala General Hospital & 4 & 4 \\
\hline \multicolumn{3}{|l|}{ Past history of mastitis in multiparous women $(N=17)$} \\
\hline No & 12 & 71 \\
\hline Yes & 5 & 29 \\
\hline \multicolumn{3}{|l|}{ Type of treatment received } \\
\hline Flucloxacillin $500 \mathrm{mg}+$ aspiration & 18 & 72 \\
\hline Flucloxacillin 500 mg + aspiration+ infiltration ceftriaxone & 6 & 24 \\
\hline Erythromycin + aspiration & 1 & 4 \\
\hline \multicolumn{3}{|l|}{ Gestational age at delivery (weeks) } \\
\hline 38 & 8 & 32 \\
\hline 39 & 8 & 32 \\
\hline 40 & 4 & 16 \\
\hline 41 & 5 & 20 \\
\hline
\end{tabular}

$3 \%$ of women with mastitis will develop lactational breast abscess [1, 2]. An incidence of lactational breast abscess ranging from 0.19 in 2007 to $0.84 \%$ in 2011 [16] has been reported, of which $70.6 \%$ were from primiparous mothers with a mean interval from delivery to breast abscess of $41.9 \pm 35.8$ days. The most frequent risk factors were sore nipples and breast engorgement [16]. The relatively low incidence in this study may be explained by the underreporting of cases of lactational breast abscess and because participants with co-morbidities (diabetes, HIV) were excluded from the study. However, since our diagnosis was mainly clinical, some cases of lactational breast abscesses may have remained undiagnosed. Furthermore, the incidence of lactational breast abscess in the present study is an estimate because the denominator used to calculate it is the number of live births (3792) in the catchment area of the three healthcare facilities during the 10 month study period. This was different from the study by Amir et al. 2004 that followed-up 1193 breastfeeding women by telephone interviews at 6 months whose denominator was exact [1]. The mean age of babies at the onset of breast abscess was $28.3 \pm 10.85$ weeks. Other studies have reported that mastitis usually occurs during the first 6 weeks but can occur at any time during lactation $[2,10]$. Mastitis is a precursor to breast abscess $[1,8]$. This is 
consistent with our study because the high mean infant age of onset of breast abscess may be associated with the weaning period of most of the participants of this study.

\section{Management of lactational breast abscess}

The traditional treatment modality for lactation breast abscesses has been I \& D in most settings in both highincome and low-income countries [17]. However, in recent times there is worldwide evidence that aspiration of lactational breast abscesses with or without ultrasound guidance has resulted in good outcomes in terms of an early resumption of breastfeeding [18, 19], cosmetic advantage, less pain and no hospitalization [8, 20,21].

Eryilmaz et al. 2005 compared a group of 22 patients with lactational breast abscess treated by aspiration without ultrasonographic guidance and another group of 23 patients treated with I \& D. They showed that in the I \& D group all patients were treated successfully, but one patient (4\%) had a recurrence 2 months after complete healing and 16 patients (70\%) in this group were not pleased with the cosmetic outcome of I \& D. In the needle aspiration group, three patients were treated with a single aspiration and 10 patients (45\%) with multiple aspirations, but nine patients (41\%) did not heal following needle aspiration and subsequently required I \& D too. No recurrences were observed in the needle aspiration group during the follow-up period. In our study six (24\%) had one aspiration, eight (32\%) had two aspirations, while $11(46 \%)$ had three aspirations. Similarly, O'Hara et al. reported $85 \%$ cure rates of 22 abscesses, some of which were aspirated without sonographic guidance especially if abscess was less than $5 \mathrm{~cm}$ on clinical examination [22]. Schwarz et al. also reported aspiration without sonographic guidance plus oral antibiotics in 33 patients with a cure rate of $82 \%$ [23]. A recent study reported that ultrasound percutaneous guided management of lactational breast abscess was successful for $96 \%$ $(102 / 105)$ of cases regardless of abscess size and allowed continued breastfeeding [20]. We treated difficult abscesses with aspiration and irrigation with saline and infiltration of ceftriaxone $1000 \mathrm{mg}$ in the abscess cavity. The rate of ceftriaxone absorption through the abscess cavity and its bioavailability is not well known. However, bacteria present in the abscess cavity are exposed to high concentrations of the antibiotic injected directly into the abscess cavity. Therefore, use of a large-bore needle, irrigation with saline solution and local infiltration of ceftriaxone appear beneficial in difficult cases because the saline reduces the viscosity of the pus thereby facilitation aspiration and also enables loculations to be disrupted whereas the antibiotic acts directly on microorganisms in high concentrations, although several aspirations were required. Further research is needed to understand the role of antibiotic infiltration in the abscess cavity. Irusen et al. [10] in a systematic review reported a mean time to resolution for women who received a course of antibiotics of 7 days, 7 days for women who received a single dose of antibiotics and 7 days for women who did not receive antibiotics. That notwithstanding, they concluded that there is insufficient evidence to determine whether needle aspiration is a more effective option to I\&D for lactational breast abscesses, or whether an antibiotic should be routinely added to women undergoing I\&D for lactational breast abscess [10].

\section{Time to resolution of breast abscess}

The resolution time of lactational breast abscesses in this study ranged from 6 to 10 days. However, several studies have reported shorter resolution time of lactational breast abscess among participants receiving treatment with needle aspiration compared with I\&D though there was a higher failure rate of treatment in the needle aspiration group [8, 24, 25].

\section{Continued breastfeeding}

In the present study, married women with lactational breast abscess continued breastfeeding for longer periods than single mothers. This is consistent with other studies that reported $87 \%$ of participants who continued breastfeeding among those treated with ultrasound-guided aspiration of lactational breast abscess [25]. Primiparous women and single breastfeeding mothers could, therefore, be targeted for extra information in preparation for parenthood classes about breast care thereby avoiding breast abscesses.

\section{Strengths and limitations of the study}

This is the first study in Cameroon, a low-income country that describes the management of lactational breast abscesses by percutaneous aspiration. This may pave the way for other studies that compare the practice of needle aspiration and I \& D in Cameroon. This study was hospital-based and only women who consulted the DGH with lactational breast abscess were enrolled for the study. Some abscesses may have been missed because we did not use ultrasonography in the management of the abscesses because it was not cost-effective for most patients. We did not include the sizes of the abscesses, although this would have enabled comparability to other studies. The incidence calculation is an estimate from the number of deliveries in the catchment area during the 10 months of study.

The participants who reported a history of lactational mastitis/abscess in previous births had no supportive medical report to affirm the diagnosis. Finally, we excluded from study women with co-morbidities (caesarean section, preterm birth, HIV-positive and diabetic 
mothers), which could increase the risk of lactational breast abscess.

\section{Conclusions}

The estimated incidence of lactational breast abscess at the Douala General Hospital is $0.74 \%$. Percutaneous needle aspiration under local anaesthesia is an effective treatment for superficial lactational abscesses in most cases with or without ultrasound guidance. It should, therefore, be recommended worldwide as first line treatment of breast abscess. Further research is needed to understand the role of local infiltration of antibiotics into the abscess cavity.

\section{Abbreviations}

CPDH: Cite des Palmier district hospital; DGH: Douala General Hospital; HIV: Human immunodeficiency virus; I \& D: Incision and drainage; IQR: Interquartile range; IWC: Infant welfare clinic; LHD: Laquintinie Hospital Douala; SPSS: Statistical Package for the Social Sciences

\section{Acknowledgements}

We are grateful to the doctors, midwives, and nurses of the DGH who assisted us during this study. We are also thankful to the study participants for giving us the information for the study.

\section{Authors' contributions}

TOE and NT conceptualized and drafted the manuscript and approved the final version of the manuscript. HE and TNN collected the data, read and approved the final version of the manuscript. TOE, NT analyzed the data. TOE, TNN, and HE conducted the needle aspiration. All the authors read and approved the final version of the manuscript.

\section{Funding}

None.

\section{Availability of data and materials}

"The datasets used and/or analyzed during the current study are available from the corresponding author on reasonable request".

\section{Ethics approval and consent to participate}

Ethical clearance was obtained from the ethics committee of the Douala General Hospital. Participants gave written informed consent.

\section{Consent for publication}

Not applicable.

\section{Competing interests}

The authors declare that they have no competing interests.

\section{Author details}

${ }^{1}$ Faculty of Health Sciences, University of Buea, Box 63, Buea, Cameroon. ${ }^{2}$ Department of Obstetrics and Gynecology, Douala General Hospital, P.O. Box 4856, Douala, Cameroon. ${ }^{3}$ Faculty of Medicine and Pharmaceutical Sciences, University of Douala, Douala, Cameroon. ${ }^{4}$ Department of Obstetrics and Gynecology, Laquintinie Hospital Douala, Douala, Cameroon.

Received: 12 April 2019 Accepted: 6 April 2020

Published online: 10 April 2020

\section{References}

1. Amir LH, Forster D, McLachlan H, Lumley J. Incidence of breast abscess in lactating women: report from an Australian cohort. BJOG. 2004;111:1378-81.

2. Amir LH, Academy of Breastfeeding Medicine Protocol Committee. ABM clinical protocol\# 4: Mastitis, revised March 2014. Breastfeed Med. 2014;9: 239-43.

3. Dixon JM, Khan LR. Treatment of breast infection. BMJ. 2011;342:d396.
4. Dabbas N, Chand M, Pallett A, Royle GT, Sainsbury R. Have the organisms that cause breast abscess changed with time?--implications for appropriate antibiotic usage in primary and secondary care. Breast J. 2010;16:412-5.

5. Dener C, Inan A. Breast abscesses in lactating women. World J Surg. 2003; 27:130-3.

6. Branch-Elliman W, Golen TH, Gold HS, Yassa DS, Baldini LM, Wright SB. Risk factors for Staphylococcus aureus postpartum breast abscess. Clin Infect Dis. 2011;54:71-7.

7. Kvist $L$, Rydhstroem $\mathrm{H}$. Factors related to breast abscess after delivery: a population-based study. BJOG. 2005;112:1070-4.

8. Eryilmaz R, Sahin M, Hakan Tekelioglu M, Daldal E. Management of lactational breast abscesses. Breast. 2005;14:375-9.

9. Naeem M, Rahimnajjad MK, Rahimnajjad NA, Ahmed QJ, Fazel PA, Owais M. Comparison of incision and drainage against needle aspiration for the treatment of breast abscess. Am Surg. 2012:78:1224-7.

10. Irusen H, Rohwer AC, Steyn DW, Young T. Treatments for breast abscesses in breastfeeding women. Cochrane Database Syst Rev. 2015;8:CD010490.

11. Chandika AB, Gakwaya AM, Kiguli-Malwadde E, Chalya PL. Ultrasound guided needle aspiration versus surgical drainage in the management of breast abscesses: a ugandan experience. BMC Res Notes. 2012;5:12.

12. Demographic Health Survey Cameroon 2011 - Google Search. https://www. google.com/search?source=hp\&ei=SMxrXcQ7htho8amrsAM\&q= demographicthealth+survey+cameroon+2011\&oq=Demographic+health+ surver+Cameroon\&gs_l=psy-ab.1.1.0i22i30l2.1205.32405.36342 ..0.0..0.560. 9767.0j3j21j5j3j2 .....0...1..gws-wiz...0i131j0j35i39j0i203j0i13j0i22i10i30. oOQZAGUEJJE. Accessed 1 Sept 2019.

13. Nlend A, Wamba G, Same Ekobo C. Infant nutrition from 0 to 36 months in Cameroon urban towns. Med D'Afrique Noire. 1997:44:47-51.

14. Chiabi A, Kamga B, Mah E, Bogne J, Nguefack S, Fokam P, et al. Breastfeeding practices in infants in the west region of Cameroon. Iran J Public Health. 2011;40:11-7.

15. General Assembly of the World Medical Association. World Medical Association Declaration of Helsinki: ethical principles for medical research involving human subjects. J Am Coll Dent. 2014;81(3):14.

16. Boccaccio C, Verdaguer Babic V, Botto L, Cervetto MM, Cetani S, Paladino S, et al. Methicillin-resistant Staphylococcus aureus (MRSA) isolation in breast abscesses in a public maternity. Medicina (Mex). 2014;74:210-5.

17. Benson MEA. Management of breast abscesses. World J Surg. 1989;13:753-6.

18. Debord M-P, Poirier E, Delgado H, Charlot M, Colin C, Raudrant D, et al. Lactational breast abscesses: do we still need surgery? J Gynecol Obstet Biol Reprod. 2016;45:307-14.

19. Hussain N, Khan I, Ahmed T, Parveen S, Malik M, Khan Ml. Comparison of the restoration of breastfeeding after percutaneous aspiration vs incision and drainage for management of breast abscess. J Liaquat Univ Med Health Sci. 2018;17:47-51

20. Colin C, Delov AG, Peyron-Faure N, Rabilloud M, Charlot M. Breast abscesses in lactating women: evidences for ultrasound-guided percutaneous drainage to avoid surgery. Emerg Radiol. 2019;26:507-14.

21. Sun H-D, Teng S-W, Huang B-S, Hsiao S-M, Yen M-S, Wang P-HP. Combination of ultrasound-guided drainage and antibiotics therapy provides a cosmetic advantage for women with methicillin-resistant Staphylococcus aureus breast abscess. Taiwan J Obstet Gynecol. 2014;53: 115-7.

22. O'Hara RJ, Dexter SPL, Fox JN. Conservative management of infective mastitis and breast abscesses after ultrasonographic assessment. Br J Surg. 1996;83:1413-4.

23. Schwarz RJ, Shrestha R. Needle aspiration of breast abscesses. Am J Surg. 2001;182:117-9.

24. Suthar K, Mewada BN, Surati K, Shah J. Comparison of percutaneous ultrasound guided needed aspiration and open surgical drainage in management of puerperal breast abscess. Int J Med Sci Public Health. 2013; 2:69-72.

25. Saleem S, Farooq T, Khan N, Shafiq M, Azeem M, Dab RH. Puerperal breast abscesses. Prof Med J. 2008:15:431-6.

\section{Publisher's Note}

Springer Nature remains neutral with regard to jurisdictional claims in published maps and institutional affiliations. 